\title{
A Novel Intelligent ARX-Laguerre Distillation Column Estimation Technique
}

\author{
Farzin Piltan ${ }^{1}$, Shahnaz TayebiHaghighi ${ }^{2}$, Somayeh Jowkar ${ }^{2,3}$, Hossein Rashidi Bod ${ }^{2}$, \\ Amirzubir Sahamijoo ${ }^{2}$ and Jeong-Seok Heo ${ }^{1, *}$ \\ ${ }^{1}$ School of IT Convergence, University of Ulsan, Ulsan, South Korea \\ ${ }^{2}$ Control and Robotic Lab, IRAN SSP Research and Development Center, Shiraz, Iran \\ ${ }^{3}$ Department of Information Technology, Faculty of Computer Engineering, \\ Ateneo De Manila University, Manila, Philippines \\ E-mail: piltanfarzin@gmail.com, heojs@ulsan.ac.kr* (*Corresponding author)
}

Received: 10 September 2018; Accepted: 05 January 2019; Published: 08 April 2019

\begin{abstract}
In practical applications, modeling of real systems with unknown parameters such as distillation columns are typically complex. To address issues with distillation column estimation, the system is identified by a proposed intelligent, auto-regressive, exogenousLaguerre (AI-ARX-Laguerre) technique. In this method, an intelligent technique is introduced for data-driven identification of the distillation column. The Laguerre method is used for the removal of input/output noise and decreases the system complexity. The fuzzy logic method is proposed to reduce the system's estimation error and to accurately optimize the ARX-Laguerre parameters. The proposed method outperforms the ARX and ARXLaguerre technique by achieving average estimation accuracy improvements of $16 \%$ and $9 \%$, respectively.
\end{abstract}

Index Terms-Distillation column, system identification, ARX modeling, intelligent nonlinear-ARX-Laguerre, ARX-Laguerre modeling.

\section{INTRODUCTION}

Process industries, and the distillation column systems used within those industries, are significantly nonlinear which creates multiple challenges in system modeling, control, and fault detection. A block diagram of a distillation column, typical in the oil and gas industries, is shown in Fig. 1. In practical applications, the uncertain conditions surrounding distillation columns results in an increase in difficulty for system modeling, control, and fault identification. However, different types of modeling have been defined for nonlinear systems. These are usually divided into numerical-mathematical and physical-mathematical modeling [1-3], which are the two primary methods for mathematics-based system modeling $[4,5]$. In practice, mathematical modeling of a distillation column is complicated due to its complexity and lack of information regarding various parameter values. To address mathematical modeling issues, a novel system identification technique is proposed.

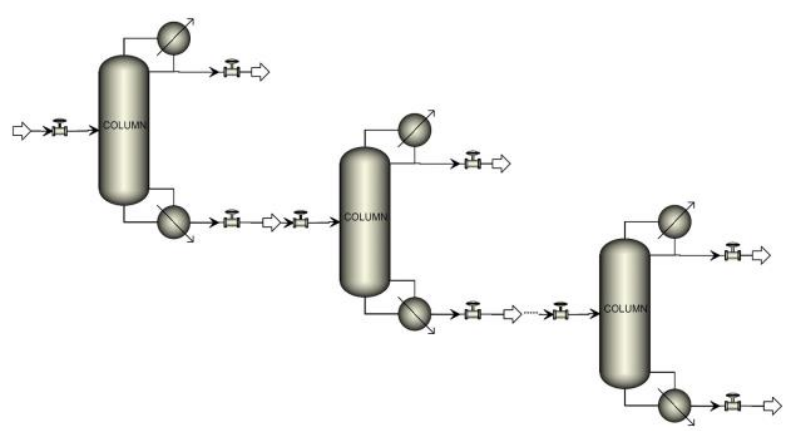

Fig.1. Continuous distillation column

There are a variety of techniques available for use in system identification, but they are primarily divided into two main groups: linear system identification techniques and nonlinear system identification techniques. Over the years, the availability of system identification techniques for modeling linear and nonlinear systems has increased. As shown in the literature [6,7], linear and nonlinear identification techniques have been introduced for numerous types of systems. Linear modeling of linear and nonlinear systems by ARX techniques has been presented to reduce the complexity of operation parameters. The linear-ARX method has been used in various applications for system identification [6,7] and fault detection [8]. While advantageous, drawbacks to the ARX method include difficulties modeling in the presence of a disturbance and/or external noise. In an attempt to solve this issue, the Laguerre filter has been used to reduce the effect of noise on the input and output [9]. As a result, the ARX-Laguerre method has been used for system identification in various applications including robot arms [10] and rolling bearing elements [11]. The primary drawback of this technique is system estimation in highly nonlinear and uncertain systems. To improve on these drawbacks, an intelligent ARX-Laguerre technique is recommended. For the first time, a nonlinear-ARX system identification method was introduced by Billings in 1980 for nonlinear-discrete system modeling [12]. Different techniques can be introduced for nonlinear- 
ARX system modeling, such as block-structured system modeling [13], neural network-based system modeling [14], and Volterra series system modeling [15]. Nonlinear-ARX system modeling has been used for the modeling of several real systems, including an aluminum plate [16], wind turbine [17], and bearing systems [18, 19]. In real-world applications, the impact of noise plays a significant role in accurate system estimation. Several researchers have introduced methods for the removal of signal noising. The ARX-Laguerre technique is a dominant method for system identification in the presence of noise and unknown conditions, and is used to reduce the model estimation complexity despite unknown conditions [20].

An intelligent ARX-Laguerre system estimation is proposed in this paper to model a distillation column, based on adaptive techniques. In the proposed method, the ARX-Laguerre parameters are modified by an adaptive artificial intelligence technique. The accuracy of the system identification is verified by ARX and ARXLaguerre techniques, with application to a distillation column. This paper has been divided into the following sections: Section two contains the related works, while the paper challenge and the proposed method objectives will be presented in Section 3. Section 4 provides the intelligent ARX-Laguerre estimation and analysis of the distillation column, results, and discussion. Section 5 concludes this paper.

\section{RELATED WORKS}

Modeling and estimation of industrial systems are significant topics of research in recent years. The distillation column is a nonlinear system, and its complexity increases when used in industry [21]. The subspace identification algorithm for distillation columns was introduced in [22]. This technique is linear, and can be useful for distillation columns with limited uncertainties. A comparative study between two types of linear system estimations, with application to the industrial electric motor, has been introduced in [6]. These two methods demonstrated acceptable performance for this system, but in the presence of noise, these two methods must be reevaluated. Li et al. [23] introduced a nonparametric method for modeling and estimation of nonlinear systems. The neural network method was recommended for analysis in that publication due to the robustness of the artificial neural network (ANN). There are various advantages to the conventional neural network technique, but the heavy computation load is a significant drawback to this technique. To improve on classical ANN, the gradient descent algorithm has been recommended to increase the system performance [24]. Further techniques, such as genetic algorithm (GA) and partial swarm optimization (PSO) have been introduced recently to improve the performance of ANN and reduce its computation load. The intelligent ARX technique has been introduced in [25], where the performance was improved by ANN and PSO. The main challenge in the use of this method is a lack of robustness. The ARX-
Laguerre method for fault identification and system estimation is introduced in $[10,11]$. Here, the ARXLaguerre method has been used for robot manipulator estimation [10] and bearing estimation [11]. However, these methods decrease the system performance in highly nonlinear systems.

\section{PROBLEM STATEMENT}

The distillation column is a nonlinear system with unknown parameters. Therefore, accurate mathematical modeling in this system in the presence of uncertainty and unknown inputs is complicated. Based on [20, 26], the system's process information is listed in Table 1. Dynamic formulation of the distillation column in the presence of uncertainty is considered using the following formulations [1]:

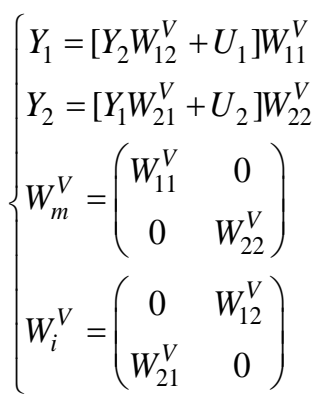

where $Y_{i}, U_{i}, W_{i}$ are output, input, and state, respectively. In a distillation column, the system inputs are heat $(E)$, the rate of reflux ratio $(\varepsilon)$ and the state, defined by $(M)$. Therefore, the system dynamics can be arranged as follows:

$$
\left[\begin{array}{l}
Y_{1} \\
Y_{2}
\end{array}\right]=\left[\begin{array}{ll}
M_{11} & M_{12} \\
M_{21} & M_{22}
\end{array}\right]\left[\begin{array}{l}
D e \\
D E
\end{array}\right]
$$

Referring to [20], the transfer function for the proposed distillation column is presented below,

$$
\left[\begin{array}{l}
Y_{1} \\
Y_{2}
\end{array}\right]=\left[\begin{array}{cc}
\frac{-1248}{1+6.9 S} & \frac{140}{(1+1.26 S)(1+1.58 S)} \\
\frac{-1590}{1+2.9 S} & \frac{344}{1+1.69 S}
\end{array}\right]\left[\begin{array}{c}
D e \\
D E
\end{array}\right]
$$

where $Y_{1}, Y_{2}$ are the differences in the two product temperatures, respectively. The frequency domain response of the distillation column is shown in Fig. 2. Based on Fig. 2, the primary harmonics in this system are 13.7 $\left(\frac{R a d}{\mathrm{sec}}\right)$ and $15.7\left(\frac{R a d}{\mathrm{sec}}\right)$. However, based on [20], the system's transfer function can be extracted from the distillation column but this scenario is very complicated. To reduce the complexity, we propose a new method. 
Table 1. Distillation Column Specifications [26]

\begin{tabular}{ll}
\hline Specification & Value \\
\hline Feed specifications & \\
Feed flow rate & $10 \mathrm{~L} / \mathrm{min}$ \\
Feed composition & 25 ethanol, 75 water, mol\% \\
Reflux flow rate & $0.6 \mathrm{~L} / \mathrm{min}$ \\
Feed temperature & $65{ }^{\circ} \mathrm{C}$ \\
Feed pressure & 1 atm \\
Reflux ratio & 2.5 molar \\
Column specifications & \\
Column height & 5.5 m \\
Distillate flow rate & $2.5 \mathrm{~L} / \mathrm{min}$ \\
Colum diameter & 0.15 m \\
Type of tray & Bubble cap tray \\
No. of trays & 15 -plates \\
Tray spacing & Approx. $35 \mathrm{~cm}$ \\
Holdup & $50 \%$ for 10 min \\
Top temperature & $83.43{ }^{\circ} \mathrm{C}$ \\
Bottom temperature & $92.75{ }^{\circ} \mathrm{C}$ \\
Reboiler duty & $183.3 \mathrm{~kW}$ \\
Condenser duty & $-167.8{ }^{\circ} \mathrm{C}$ \\
Column pressure & 1 atm \\
Distillate composition & 85 ethanol, 16 water, mol\% \\
Bottom composition & 17 ethanol, 83 water, mol\% \\
\hline & \\
\hline &
\end{tabular}

This paper introduces the following problems, as well as brief solutions.

Problem 1: Extracting the dynamic, accurate mathematical formulation of the distillation column is complicated.

Solution 1: The ARX identification technique is recommended for system estimation based on the input/output signals.

Problem 2: In the presence of external disturbances, the accuracy of the ARX estimation technique can be reduced.

Solution 2: The ARX-Laguerre technique is recommended for removing noise from the signal input and output.

Problem 3: System complexity is reduced using the ARX-Laguerre technique when compared to the ARX technique, but the system complexity is still high.

Solution 3: To solve this challenge, this research introduces an intelligence-based ARX-Laguerre method .

\section{Proposed Methodology}

To effectively model the distillation column using a signal-based system identification technique, an adaptive intelligence-based ARX-Laguerre technique is recommended. In the first step, system modeling based on the ARX method is proposed.
ARX System Estimation: An extensive variety of distillation columns can be specified using ARX techniques [27]:

$$
\begin{aligned}
& Q(\kappa)+\ell_{1} Q(\kappa-1)+\ell_{2} Q(\kappa-2)+\ldots+\ell_{w} Q\left(\kappa-n_{q}\right)= \\
& \varepsilon_{1} I(\kappa-1)+\varepsilon_{2} I(\kappa-2)+\ldots+\varepsilon_{i} I\left(\kappa-n_{i}\right)+e(\kappa)
\end{aligned}
$$

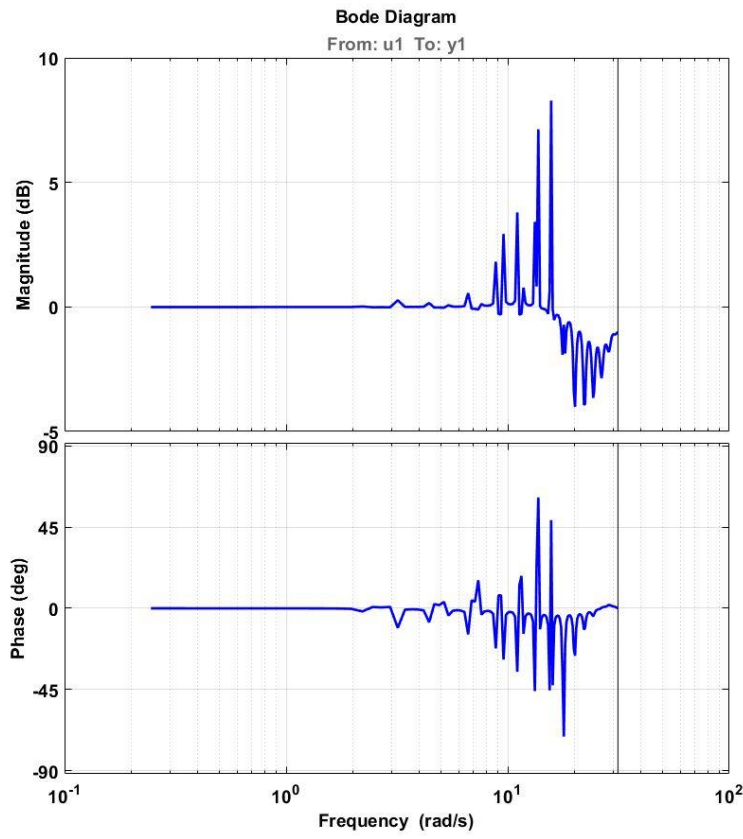

Fig.2. Frequency domain response

Based on (4), the future output can be predicted by using previous observation parameters,

$$
\begin{aligned}
& Q(\kappa)=-\ell_{1} Q(\kappa-1)-\ldots-\ell_{q} Q\left(\kappa-n_{q}\right)+ \\
& \varepsilon_{1} I(\kappa-1)+\ldots+\varepsilon_{i} I\left(\kappa-n_{i}\right)+e(\kappa)
\end{aligned}
$$

where $\left(Q\left(\kappa-n_{q}\right), I\left(\kappa-n_{i}\right)\right), Q(\kappa), I(\kappa),\left(\ell_{*}, \mathcal{E}_{*}\right), e(\kappa) \quad$ are the systems lag, output, input, unknown parameters, and zero-mean noise, respectively. The state-space system is defined as follows:

$$
\left\{\begin{array}{l}
Q(\kappa)=-\varpi(\kappa) \cdot \chi+e(\kappa) \\
\chi=\left[\begin{array}{llll}
\ell_{1} \ldots & \ell_{q} & \varepsilon_{1} \ldots & \varepsilon_{i}
\end{array}\right] \\
\varpi=\left[\begin{array}{llll}
-Q(\kappa-1) \ldots & -Q\left(\kappa-n_{w}\right) & I(\kappa-1) \ldots & I\left(\kappa-n_{i}\right)
\end{array}\right]
\end{array}\right.
$$

Here, $\varpi$ is a regressor variable of ARX modeling and $\chi$ is the unknown parameters matrix. According to (5) and (6) the ARX system estimation is defined as (7).

$$
\begin{aligned}
& Q(\kappa)=\bar{\theta}\left[Q(\kappa-1), Q(\kappa-2), \ldots, Q\left(\kappa-n_{w}\right)\right. \\
& \left., I(\kappa-1), I(\kappa-2), \ldots, I\left(\kappa-n_{i}\right)\right]
\end{aligned}
$$

The least-squares technique has been defined to estimate and predict the best value for $\bar{\theta}$. 


$$
\begin{aligned}
& \bar{\theta}^{*}=\arg \min _{\bar{\theta}}\{f(\bar{\theta}, \partial)\} \\
& f(\bar{\theta}, \partial)=\frac{1}{k} \sum_{0}^{k}(Q(\kappa)-\varpi(\kappa) \chi)^{2}
\end{aligned}
$$

Fig. 3 illustrates the estimation performance for the distillation column based on the ARX techniques. According to the figure, the fit criterion for ARX is about $74.2 \%$ for this system.

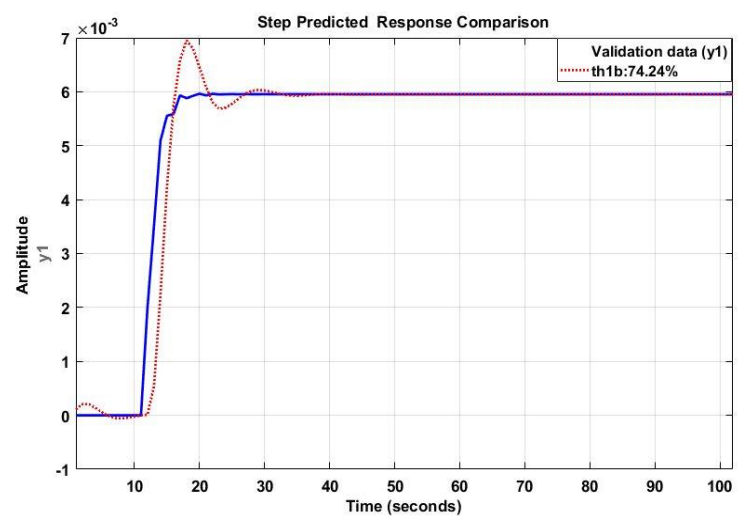

Fig.3. Fifth order ARX estimation with application to a distillation column

After system estimation using the ARX technique, an alternate technique can be used for system modeling, such as an IDPOLY, Z-transform, state-space, or transfer function. In this paper, transfer function system modeling is used for both modeling and analysis. The discrete time ARX model is calculated as follows:

$$
\begin{aligned}
& A(z) Y(t)=B(z) U(t)+e(t) \\
& A(z)=1+Z^{-1}+6 Z^{-2}+Z^{-3}-3 Z^{-4}-7 Z^{-5} \\
& B(z)=-0.3-0.8 Z^{-1}-3 Z^{-2}-6 Z^{-3}-7 Z^{-4}
\end{aligned}
$$

Based on Fig. 3 and (9), the transfer function of the ARX model is obtained by the following:

$$
H=\frac{-0.3 S^{4}-0.8 S^{3}-3 S^{2}-6 S-7}{S^{5}+S^{4}+6 S^{3}+S^{2}-3 S-7}
$$

Based on (10), this system is modeled by a $5^{\text {th }}$ order equation. The classical ARX technique has two significant drawbacks: the estimation accuracy (74.24\%) and the high system estimation order (5th order), causing highly nonlinear behavior. To address these issues, the ARX-Laguerre method is introduced in the next step.

ARX-Laguerre System Estimation: The state-space function based on ARX system estimation is calculated as follows:

$$
\left\{\begin{array}{l}
\left.S(\kappa+1)=\left[A S(\kappa)+b_{i} I(\kappa)\right]+\Delta_{d}(\kappa)\right] \\
Q(\kappa)=(\alpha)^{T} S(\kappa)
\end{array}\right.
$$

where $S(k),\left(A, b_{i}, \alpha\right), Q(k), I(k), \Delta_{d}(k)$ are the state of the system, unknown parameters, output, input, and system uncertainty, respectively. Regarding (10), the future state and current output of the system depend on two factors: current states and current input. To improve accuracy, performance, and to reduce the system's estimation order, the ARX-Laguerre technique is proposed,

$$
\begin{aligned}
& Q(\kappa)=\sum_{0}^{N_{\alpha}-1} K_{n, \alpha}\left(\sum_{j=1}^{\infty} \frac{\sqrt{1-\zeta_{\alpha}^{2}}}{Z-\zeta_{\alpha}}\left(\frac{1-\zeta_{\alpha}, z}{Z-\zeta_{\alpha}}\right)^{n} * Q(\kappa)\right) \\
& S_{n, q}(\kappa)+\sum_{0}^{N_{\beta}-1} K_{n, \beta}\left(\sum_{j=1}^{\infty} \frac{\sqrt{1-\zeta_{\beta}^{2}}}{Z-\zeta_{\beta}}\left(\frac{1-\zeta_{\beta}, z}{Z-\zeta_{\beta}}\right)^{n} *\right. \\
& I(\kappa)) . S_{n, I}(\kappa)
\end{aligned}
$$

where $\left(K_{n, \alpha} \& K_{n, \beta}\right) \quad, \quad\left(N_{\alpha} \& N_{\beta}\right) \quad, *, \quad S_{n, q}(k)$, $S(k),\left(\left(\frac{\sqrt{1-\xi_{\alpha}^{2}}}{z-\xi_{\alpha}} \cdot \frac{1-\xi_{\alpha}^{2}, z^{n}}{z-\xi_{\alpha}}\right),\left(\frac{\sqrt{1-\xi_{\beta}^{2}}}{z-\xi_{\beta}} \cdot \frac{1-\xi_{\beta}^{2}, z^{n}}{z-\xi_{\beta}}\right)\right)$ are the Fourier coefficients, system order, convolution product, filterbased input signal, state signal, and Lagrange base function, respectively. According to (12), the state-space equation for the ARX-Laguerre system estimation is proposed as follows.

$$
\left\{\begin{array}{l}
\left.S(\kappa+1)=\left[A S(\kappa)+b_{i} I(\kappa)+b_{q} Q(\kappa)\right]+\Delta_{d}(\kappa)\right] \\
Q(\kappa)=(\alpha)^{T} S(\kappa)
\end{array}\right.
$$

Based on (12), the future state in the ARX-Laguerre system estimation technique is related to the current state, input, and output. This technique reduces the system's order estimation. Fig. 4 demonstrates the estimation accuracy for modeling based on the ARX-Laguerre method. Based on Fig. 4 and (13), the system estimation for the proposed system is calculated as follows:

$$
\begin{aligned}
& \left\{\begin{array}{l}
\left.S(\kappa+1)=\left[A S(\kappa)+b_{i} I(\kappa)+b_{q} Q(\kappa)\right]+\Delta_{d}(\kappa)\right] \\
Q(\kappa)=(\alpha)^{T} S(\kappa)
\end{array}\right. \\
& A=\left[\begin{array}{cc}
0 & 1 \\
-1.87 & -1
\end{array}\right], b_{i}=\left[\begin{array}{l}
1 \\
1
\end{array}\right], b_{q}=\left[\begin{array}{ll}
0.015 & 0.26
\end{array}\right] \\
& \alpha=\left[\begin{array}{ll}
0.025 & 0.26
\end{array}\right]
\end{aligned}
$$

Based on (14), the transfer function for ARX-Laguerre distillation column estimation is calculated as follows:

$$
H=\frac{0.2 S+0.4}{S^{2}+0.5 S+0.4}
$$

Regarding Figure 4, the fit criterion for the ARXLaguerre system is shown to be approximately $80 \%$. To further improve the performance of ARX and ARXLaguerre system estimations, the AI-ARX-Laguerre method is proposed. 


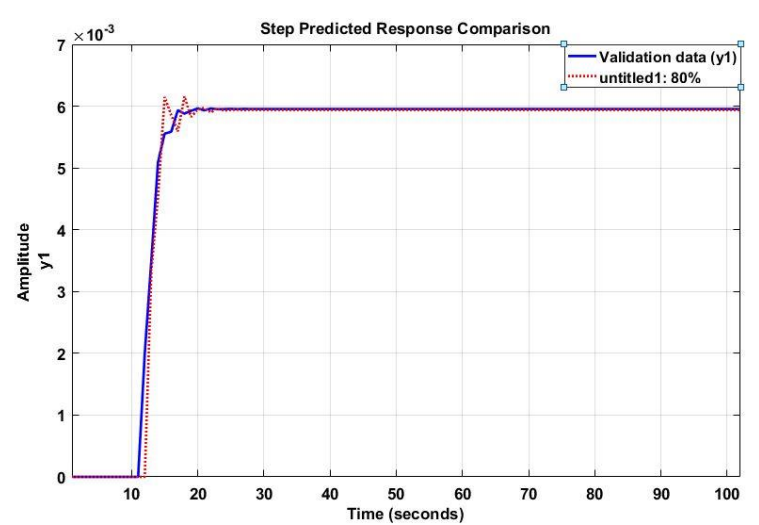

Fig.4. Second order ARX-Laguerre estimation with application to a distillation column

AI ARX-Laguerre System Estimation: The ARXLaguerre technique reduced the ARX system order from $5^{\text {th }}$ to $2^{\text {nd }}$ order, and increased the fit criterion from $74.24 \%$ to $80 \%$. To increase the system's modeling performance, the AI ARX-Laguerre technique is introduced here. The intelligent function is defined in the following equation,

$$
\begin{aligned}
& Q(\kappa)=f_{A I}\left[Q(\kappa-1), Q(\kappa-2), \ldots, Q\left(\kappa-n_{q}\right)\right. \\
& \left., I(\kappa-1), I(\kappa-2), \ldots, I\left(\kappa-n_{i}\right)\right]
\end{aligned}
$$

where $f_{A I}$ is a nonlinear function for system estimation, defined by the intelligent technique. The nonlinear intelligent function, $f_{A I}$, is calculated using the following equation:

$$
\begin{aligned}
& Q(\kappa)=\Omega_{0}+\sum_{i i=1}^{n} \Omega_{i i_{1}} S_{i i_{1}}(\kappa)+ \\
& \sum_{i i_{1}=1}^{n} \sum_{i i=1}^{n} \Omega_{i i_{1}} \Omega_{i i_{2}} S_{i i_{1}}(\kappa) S_{i i_{2}}(\kappa)+\ldots
\end{aligned}
$$

where $\Omega_{i}$ is the coefficient for system modeling, $n=n_{q}+n_{i}$ and $S_{i}(\kappa)$ is:

$$
S_{i}(\kappa)=\left\{\begin{array}{cc}
Q(\kappa-i i) & 1 \leq i i \leq n_{q} \\
I\left(\kappa-\left(i i-n_{q}\right)\right. & n_{q}+1 \leq 1 \leq n_{q}+n_{i}
\end{array}\right.
$$

Therefore, based on the (17) and (18) we have

$$
Q(\kappa)=\sum_{\hbar=1}^{\frac{(n n+r r) !}{(r r !)(n n !)}} \chi_{\hbar} \Phi_{\hbar}(\kappa)
$$

where $\chi_{\hbar}$ is the coefficient of system modeling, and $\Phi_{\hbar}(k)$ is a regression term. The orthogonalization model is calculated as the follows:

$$
Q(\kappa)=\left[\Phi_{1}, \Phi_{2}, \ldots, \Phi_{\hbar}\right] \times\left[\Omega_{1}, \Omega_{2}, \ldots, \Omega_{\hbar}\right]
$$

Therefore, based on (19) and (20), the Schmidt's orthogonal formulation is expressed as

$$
\begin{aligned}
& Q(k)=\Phi \times \Omega=\vartheta \times \eta \\
& \vartheta=\Phi_{\hbar}-\sum_{\hbar=1}^{\frac{(n n+r r) !}{(r r !)(n n !)}-1} \frac{\left\langle\Phi_{\hbar}, \vartheta_{r}\right\rangle}{\left\langle\vartheta_{r r}, \vartheta_{r r}\right\rangle} \vartheta_{r r}, \\
& \eta=\frac{\left\langle Q, \vartheta_{\hbar}\right\rangle}{\left\langle\vartheta_{\hbar}, \vartheta_{\hbar}\right\rangle}
\end{aligned}
$$

where $\vartheta,(n n, r r), \eta$ are the orthogonal matrix, function order, and coefficient term, respectively. The error value is given as

$$
E r r=\frac{(\eta)^{2}\left\langle\vartheta_{r r}, \vartheta_{r r}\right\rangle}{\langle Q, Q\rangle} \times 100 \%
$$

Therefore, based on (21), the model structure is satisfied if we introduce the following condition:

$$
1-\sum_{\hbar=1}^{\frac{(n n+r r) !}{(r r !)(n n !)}}\{E r r\} \leq \varepsilon
$$

where $\varepsilon$ is a threshold value. Referring to (19) and (23), the orthogonal nonlinear ARX-Laguerre model is written as follows:

$$
\begin{gathered}
Q_{n}(\kappa)=\sum_{\substack{\hbar^{\prime} h^{\prime}=1 \\
\left(n^{\prime} n^{\prime}+r^{\prime} r^{\prime}\right) !}}^{\frac{\left(n^{\prime} n^{\prime}+r^{\prime} r^{\prime}\right) !}{\left(r^{\prime} r^{\prime} !\right)\left(n^{\prime} n^{\prime} !\right)}} \tilde{\lambda}_{\hbar} \tilde{\Phi}_{\hbar}(\kappa) \rightarrow \\
Q_{n}(\kappa)=\sum_{\left.\hbar^{\prime} h^{\prime} !\right)\left(n^{\prime} n^{\prime} !\right)}^{\eta_{\hbar}=1} \vartheta_{\hbar} \tilde{\psi}_{\hbar}(\kappa)
\end{gathered}
$$

where,

$$
\tilde{\psi}_{\hbar}=\tilde{\Phi}_{\hbar}-\sum_{\hbar=r r+1}^{\frac{\left(n^{\prime} n^{\prime}+r^{\prime} r^{\prime}\right) !}{\left(r^{\prime} r^{\prime} !\right)\left(n^{\prime} n^{\prime} !\right)}} \frac{\left.\vartheta_{\hbar}, \tilde{\lambda}_{\hbar}\right\rangle}{\left\langle\tilde{\lambda}_{\hbar}, \tilde{\lambda}_{\hbar}\right\rangle} \tilde{\eta}_{r r}
$$

To validate the model, different techniques have been used, such as the one step ahead method and model predicted output technique. Based on the one step ahead technique, the AI ARX-Laguerre technique is defined as follows:

$$
\begin{aligned}
& Q(\kappa)=a_{1} Q(\kappa-1)+a_{2} Q(\kappa-2) \\
& +c I(\kappa-1) I(\kappa-1)
\end{aligned}
$$


where $a_{1}, a_{2}$, and $c$ are output/input system order coefficients. Thus, the model prediction is given as:

$$
\hat{Q}(3)=a_{1} Q(2)+a_{2} Q(1)+c I(2) Q(2)
$$

The system prediction frequency response is illustrated in Fig. 5.

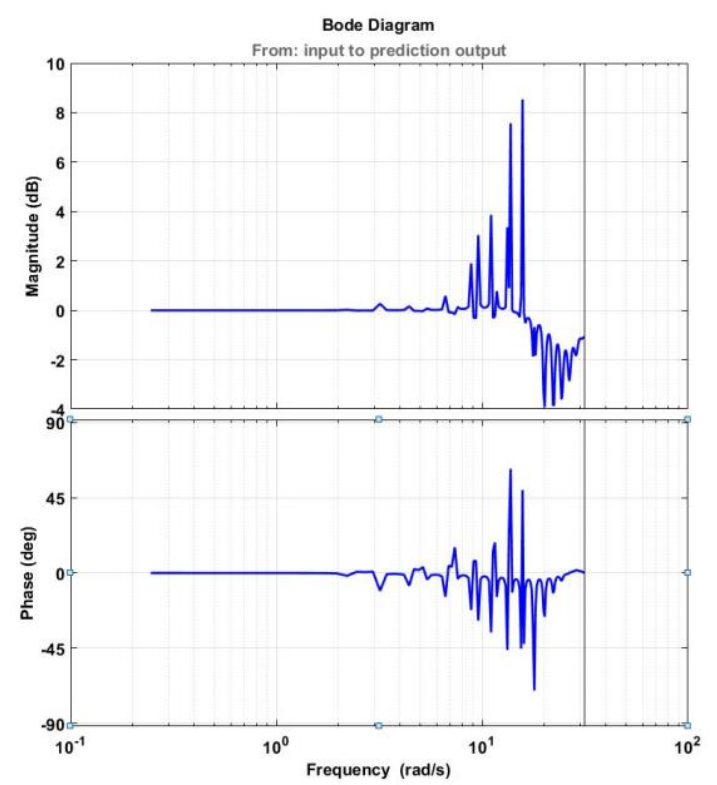

Fig.5. System prediction frequency domain response for the AI ARX-Laguerre technique

Based on Fig. 5, the primary harmonics in this system are $13.7\left(\frac{\mathrm{Rad}}{\mathrm{sec}}\right)$ and $15.7\left(\frac{\mathrm{Rad}}{\mathrm{sec}}\right)$. Based on a comparison between Fig. 2 and Fig. 5, it is clear that one step can accurately predict the frequency response of the AI-ARXLaguerre model ahead technique. The block diagram of system estimation based on the AI ARX Laguerre technique is illustrated in Fig.6.

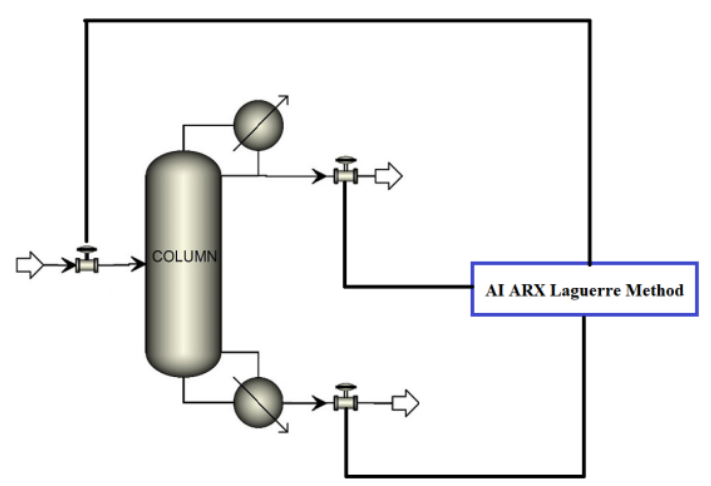

Fig.6. Block diagram of proposed AI ARX-Laguerre distillation column estimation

Fig. 7 illustrates the real step response and identified system response based on the AI ARX-Laguerre system identification technique. Regarding this figure, the system identification accuracy is approximately $89.2 \%$. Based on this method, the discrete AI ARX-Laguerre model for the distillation column is identified as:

$$
\begin{aligned}
& \Gamma(z) \hat{Q}(\kappa)=\Pi(z) I(\kappa-1)+e(\kappa-1) \\
& \Gamma(z)=1+0.1717 Z^{-1}+0.6374 Z^{-2} \\
& \Pi(z)=1.449 Z^{-1}+0.3594 Z^{-2}
\end{aligned}
$$

The root mean square error (RSME) is given as:

$$
R M S E=\sqrt{\frac{\sum_{r r=1}^{N N}\left(\hat{Q}_{r r}-Q_{r r}\right)^{2}}{N N}}
$$

Based on (28) and (29), the root mean square error is $6.588 \times 10^{-9}$.

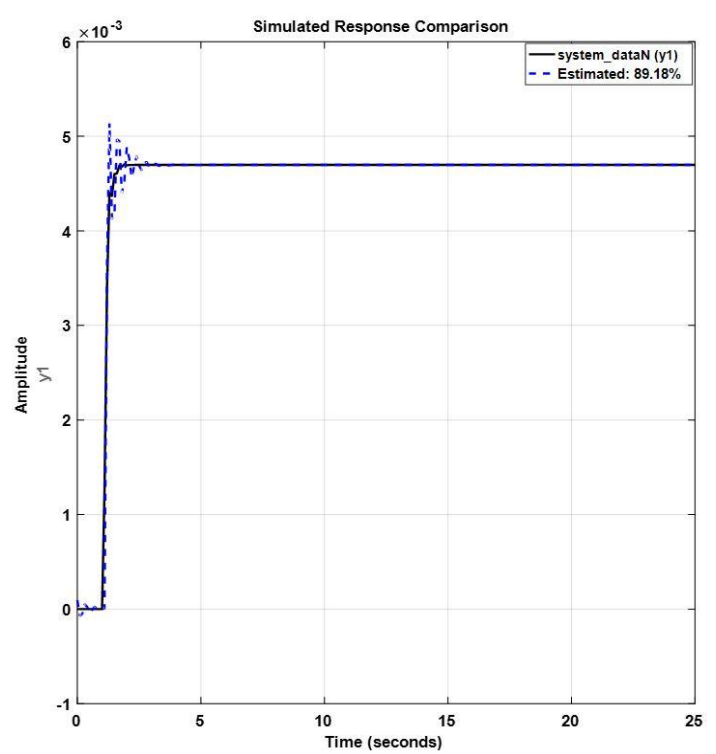

Fig.7. AI ARX-Laguerre distillation column identification technique

The distillation column estimated by the AI ARXLaguerre technique is modelled based on (30).

$$
\begin{aligned}
& Q(\kappa)=\sum_{\hbar^{\prime}=1}^{9} \tilde{\chi}_{\hbar} \tilde{\Phi}_{\hbar}(\kappa) \rightarrow \\
& Q(\kappa)=0.7986 Q(\kappa-1)+0.232 Q(\kappa-2) \\
& +0.0127 Q(\kappa-3)-0.0496 Q(\kappa-6)- \\
& 3.14 \times 10^{-4} I(\kappa-1)+7.517 \times 10^{-4} I(\kappa-2)+ \\
& 4.92 \times 10^{-4} I(\kappa-5)+0.3947 Q(\kappa-1) I(\kappa-1)+ \\
& 0.2564 Q(\kappa-2) I(\kappa-5)
\end{aligned}
$$

Based on (30), the ARX-Laguerre transfer function is calculated as follows:

$$
H=\frac{14.77 S+309.4}{S^{2}+11.24 S+309.4}
$$


The transfer function identification accuracy is represented in Fig.8. Regarding this figure, the accuracy rate is more than $91 \%$. Fig. 9 depicts the impulse response for distillation column identification by the AI ARX-Laguerre approach.

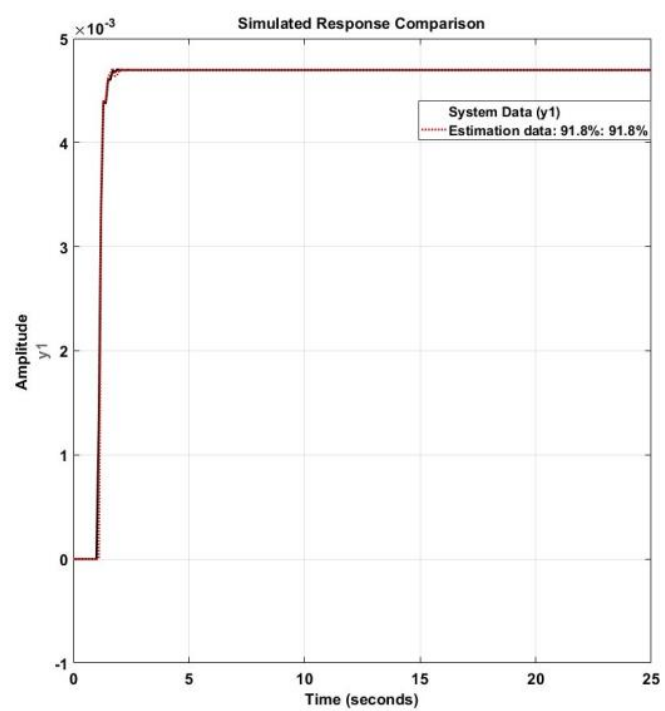

Fig.8. Continuous time identified transfer function by the AI-ARXLaguerre distillation column identification technique

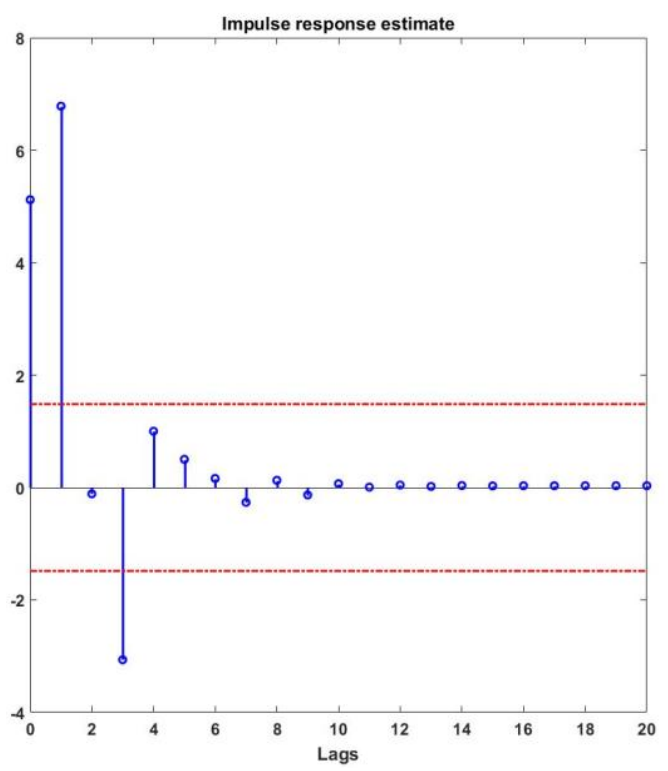

Fig.9. Estimated impulse response by the AI-ARX-Laguerre distillation column identification technique

The discrete time state-space model estimation based on the AI-ARX-Laguerre technique can be identified as

$$
\begin{aligned}
& S\left(t+T_{s}\right)=h_{11} \cdot S(t)+h_{12} \cdot I(t)+h_{21} Q(t)+K_{11} \cdot e(t) \\
& Q(t)=h_{22} \cdot S(t)+h_{23} \cdot I(t)+e(t)
\end{aligned}
$$

where $h_{11},\left(h_{12}+h_{21)}, K_{11}, h_{22}, h_{23}\right.$ are calculated as follows, respectively.

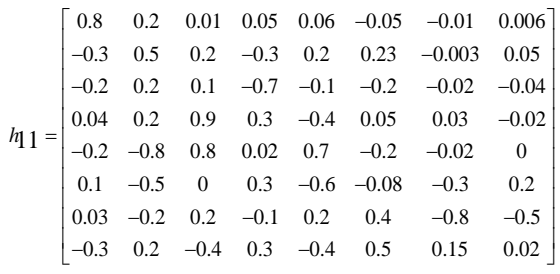

$$
\left(h_{12}+h_{21}\right)=\left[\begin{array}{c}
-3.14 \times 10^{9} \\
7.52 \times 10^{9} \\
-4.93 \times 10^{9} \\
9.74 \times 10^{9} \\
4.92 \times 10^{9} \\
-9.35 \times 10^{9} \\
5.22 \times 10^{9} \\
1.12 \times 10^{9}
\end{array}\right]
$$

$$
h_{22}=\left[\begin{array}{c}
-1.8 \times 10^{-10} \\
5.6 \times 10^{-11} \\
-4.4 \times 10^{-12} \\
1.5 \times 10^{-11} \\
-5.3 \times 10^{-12} \\
-8.1 \times 10^{-12} \\
-1.7 \times 10^{-12} \\
3 \times 10^{-12}
\end{array}\right]^{T}
$$

$$
h_{23}=[0]
$$

$$
K_{11}=\left[\begin{array}{c}
-9.53 \times 10^{8} \\
9.57 \times 10^{8} \\
-4.19 \times 10^{8} \\
1.3 \times 10^{9} \\
-1.6 \times 10^{9} \\
5.75 \times 10^{6} \\
-3.83 \times 10^{9} \\
2.8 \times 10^{9}
\end{array}\right]
$$

\section{CONCLUSION}

Mathematical models are pivotal in the analysis and design of distillation columns, but accurate modeling of the physical system is complex. Therefore, in this research, the artificial intelligence based-ARX-Laguerre 
method was introduced for distillation column modeling. The results indicate that the proposed method can improve the performance and accuracy of nonlinear distillation column modeling. Compared with ARX and ARX-Laguerre system estimations, the proposed method demonstrated improved reliability in time and frequency response. Furthermore, this technique provides a reliable model for system control, system analysis, and fault diagnosis of the distillation column.

\section{ACKNOWLEDGMENT}

This work was supported by Research Fund of University of Ulsan.

\section{REFERENCES}

[1] Bîldea, Costin S., Cătălin Pătruț, Sten Bay Jørgensen, Jens Abildskov, and Anton A. Kiss. "Cyclic distillation technology-a mini - review." Journal of Chemical Technology \& Biotechnology 91, no. 5 (2016): 1215-1223.

[2] Yang, Ruey-Jen, Chan-Chiung Liu, Yao-Nan Wang, HuiHsiung Hou, and Lung-Ming Fu. "A comprehensive review of micro-distillation methods." Chemical Engineering Journal 313 (2017): 1509-1520.

[3] Acharya, Pratima, Geetanjali Dumpa, and Tarun Kumar Dan. "Modelling and control of distillation column." In Computation of Power, Energy Information and Commuincation (ICCPEIC), 2016 International Conference on, pp. 123-128. IEEE, 2016.

[4] Nowak, Robert D. "Nonlinear system identification." Circuits, Systems and Signal Processing 21, no. 1 (2002): 109-122.

[5] Distefano, G. P. "Mathematical modeling and numerical integration of multicomponent batch distillation equations." AIChE Journal 14, no. 1 (1968): 190-199.

[6] Najeh, Tawfik, et al. "New methods of Laguerre pole optimization for the ARX model expansion on Laguerre bases." ISA transactions 70 (2017): 93-103.

[7] Vidal, René, Yi Ma, and S. Shankar Sastry. "Hybrid System Identification." In Generalized Principal Component Analysis, pp. 431-451. Springer, New York, NY, 2016.

[8] Najeh, Tawfik, Chakib Ben Njima, Tarek Garna, and José Ragot. "Input fault detection and estimation using PI observer based on the ARX-Laguerre model." The International Journal of Advanced Manufacturing Technology 90, no. 5-8 (2017): 1317-1336.

[9] Bouzrara, Kais, Tarek Garna, José Ragot, and Hassani Messaoud. "Decomposition of an ARX model on Laguerre orthonormal bases." ISA transactions 51, no. 6 (2012): 848-860.

[10] Abdelwahed, Imen Ben, Abdelkader Mbarek, and Kais Bouzrara. "Adaptive MPC based on MIMO ARXLaguerre model." ISA transactions 67 (2017): 330-347.

[11] Mbarek, Abdelkader, et al. "Laguerre-based modelling and predictive control of multi-input multi-output systems applied to a communicating two-tank system (CTTS)." Transactions of the Institute of Measurement and Control 39.5 (2017): 611-624.

[12] Leontaritis, I. J., and Stephen A. Billings. "Input-output parametric models for non-linear systems part I: deterministic non-linear systems." International journal of control 41, no. 2 (1985): 303-328.
[13] Xia, Xin, Jianzhong Zhou, Jian Xiao, and Han Xiao. "A novel identification method of Volterra series in rotorbearing system for fault diagnosis." Mechanical systems and signal processing 66 (2016): 557-567.

[14] Harnischmacher, Gerrit, and Wolfgang Marquardt. "Nonlinear model predictive control of multivariable processes using block-structured models." Control Engineering Practice 15, no. 10 (2007): 1238-1256.

[15] Arnaiz-González, Álvar, Asier Fernández-Valdivielso, Andres Bustillo, and Luis Norberto López de Lacalle. "Using artificial neural networks for the prediction of dimensional error on inclined surfaces manufactured by ball-end milling." The International Journal of Advanced Manufacturing Technology 83, no. 5-8 (2016): 847-859.

[16] Peng, Z. K., Z. Q. Lang, C. Wolters, S. A. Billings, and K. Worden. "Feasibility study of structural damage detection using NARMAX modelling and Nonlinear Output Frequency Response Function based analysis." Mechanical Systems and Signal Processing 25, no. 3 (2011): 1045-1061.

[17] Liu, Xiong, Cheng Lu, Shi Liang, Ajit Godbole, and Yan Chen. "Vibration-induced aerodynamic loads on large horizontal axis wind turbine blades." Applied Energy 185 (2017): 1109-1119.

[18] Tang, Hao, Y. H. Liao, J. Y. Cao, and Hang Xie. "Fault diagnosis approach based on Volterra models." Mechanical Systems and Signal Processing 24, no. 4 (2010): 1099-1113.

[19] JIANG, Jing, Zhinong LI, and Gaosong TANG. "Fault Diagnosis Method Based on Volterra Kernel Identification for Rotor Crack." Machine Tool \& Hydraulics 23 (2010): 040.

[20] Benabdelwahed, Imen, et al. "Nonlinear system modelling based on NARX model expansion on Laguerre orthonormal bases." IET Signal Processing 12.2 (2017): 228-241.

[21] Bachnas AA, Tth R, Ludlage JHA, Mesbah A A review on data-driven linear parameter-varying modeling approaches: a high-purity distillation column case study. $\mathbf{J}$ Process Control, 24 (2014): 272-285.

[22] Meidanshahi, Vida, et al. "Subspace model identification and model predictive control based cost analysis of a semicontinuous distillation process." Computers \& Chemical Engineering 103 (2017): 39-57.

[23] Li X, Lu WF, Zhai L et al Remaining life prediction of cores based on data-driven and physical modeling methods. In: Nee AYC (ed) Handbook of manufacturing engineering and technology. Springer, London, (2015): 3239-3264.

[24] Mavrovouniotis M, Yang S Training neural networks with ant colony optimization algorithms for pattern classification. Soft Comput 19 (2015):1511-1522.

[25] Jaleel, E. Abdul, and K. Aparna. "Identification of realistic distillation column using hybrid particle swarm optimization and NARX based artificial neural network." Evolving Systems (2018): 1-18.

[26] Taqvi, Syed A., et al. "Fault detection in distillation column using NARX neural network." Neural Computing and Applications (2018): 1-17.

[27] Wei, Hua-Liang, and Stephen A. Billings. "Model structure selection using an integrated forward orthogonal search algorithm assisted by squared correlation and mutual information." International Journal of Modelling, Identification and Control 3, no. 4 (2008): 341-356. 


\section{Authors' Profiles}

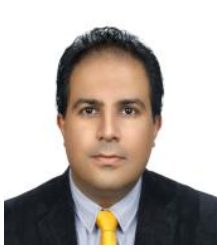

Farzin Piltan is a research associate in the department of electrical and computer engineering, at the University of Ulsan, Ulsan, South Korea. He is also a senior researcher and the president of the IRANSSP research and development center, Shiraz, Iran. He has published more than 170 peer reviewed research articles and nine books. He is also an editorial board member of four academic journals. His research interests include fault diagnosis, nonlinear control, system modeling, and embedded systems.

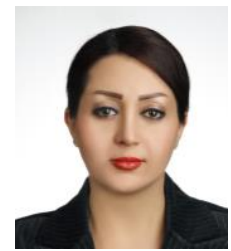

Shahnaz Tayebihaghighi is a research associate at the IRANSSP research and development center. She has published nine peer reviewed research articles and three books. Her research areas are nonlinear model-reference control, artificial intelligence, and system modeling.

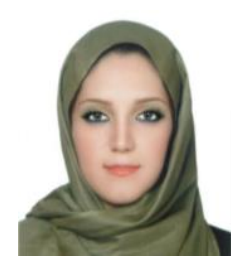

Somayeh Jowkar is a research associate at the IRANSSP research and development center.

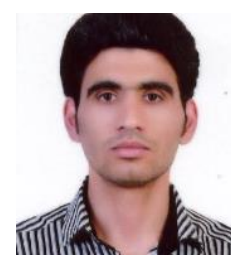

Hossein Rashidi Bod is a research student at the Institute of Advanced Science and Technology, Research Center, IRAN SSP.

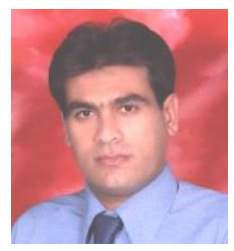

Amirzubir Sahamijoo is a research assistant at the Institute of Advanced Science and Technology, Research Center, IRAN SSP.

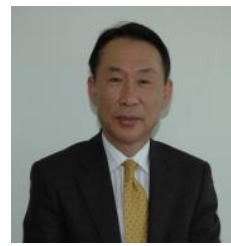

Jeong-Seok Heo is currently a professor with the Department of IT convergence and also president of Ulsan College. 